\title{
A METHOD OF STUDYING ELECTRODE POTENTIALS AND POLARIZATION ${ }^{\circ}$
}

\author{
By H. D. Holler
}

\section{ABSTRACT}

A resistance-coupled electron-tube amplifier was used to operate an oscillograph for observing and recording the variations in potential of several electrodes without requiring any current from the electrode under investigation. By superposing alternating current upon the electrode it was possible with the use of a separately excited wattmeter to measure the potential due to resistance, and compute the boundary resistance at the electrode. The paper is limited to a description of the method and illustrations of its use in the case of some typical electrodes, including both smooth and platinized platinum, carbon, lead, and copper in sulphuric acid, and copper in a copper sulphate solution.

\section{CONTENTS}

I. Introduction

II. Description of the amplifier and its use with the oscillograph____-_ 157

III. Use of the amplifier in the measurement of the resistance at an electrode

IV. Conclusions

\section{INTRODUCTION}

In any electrochemical reaction the potential difference between the electrode and the electrolyte is an important factor. The measurement of electrode potential ${ }^{1}$ is, therefore, one of the most common means of studying electrochemical processes.

The usual method of determining the potential of an electrode consists in measuring the difference in electric potential between the electrode in question and some reference electrode of constant potential, both being in electrolytic contact. With no current flowing, such a measurement with a potentiometer gives the true electromotive force of the cell and the electrode potential may be expressed in terms of the measured value of the cell, using the potential of the

$a$ The results of this investigation were presented at the Ithaca meeting of the American Chemical Society in September, 1924.

1 Following common practice in the literature on this subject, the term "potential" as used throughout this paper really means a "difference in potential." 
reference electrode as an arbitrary zero. If a continuous current be allowed to flow between the electrolyte and electrode in question, the electrode potential as measured by a potentiometer will usually be considerably different from that at zero current. This difference consists partly of an electromotive force opposing the flow of current, referred to as "polarization," and partly of a potential difference required to overcome resistance. ${ }^{2}$ This resistance includes that of the electrode material, that of the electrolyte between the reference electrode and the electrode being studied, and any resistance at the boundary between the electrode and electrolyte. The resistance between the electrode and electrolyte has been called "transfer resistance" by Newbery ${ }^{3}$ and others, but its existence has been questioned by numerous investigators. ${ }^{4}$ The measurement of decomposition voltage and overvoltage by the direct potentiometer method during the flow of a continuous current is, therefore, subject to an error equal to the potential difference required to overcome the resistance above mentioned. For convenience, this resistance is referred to throughout this paper as "boundary resistance."

The most common method of measuring the electromotive force of polarization consists in suddenly interrupting the current and observing the difference in potential across the cell immediately after the interruption. Since the difference in potential at this moment is usually a rapidly decreasing quantity, the observed value depends upon the time elapsing between the interruption of the current and the observation, and the rate at which the quantity is changing. Because of these factors the so-called commutator method. of measuring polarization must be used with great care to obtain reliable results. The observed value by this method as read by a voltmeter or potentiometer gives an average value of a variable quantity over an interval of time, depending upon the speed of the commutator, and this average value must obviously be somewhat lower than the polarization at the instant of interruption of the current.

The oscillograph was used by LeBlanc ${ }^{5}$ and Reichenstein ${ }^{6}$ for the study of polarization, but the objection to its use is obvious for the reason that considerable current is required to operate this device, and, as used, the current was necessarily supplied by the cell under investigation. The difference in potential across the cell was doubtless affected by the delivery of the current. However, since the polarization, after the interruption of the current is a rapidly

\footnotetext{
2 Lewis and Randall, "Thermodynamics," p. 386; 1923.

3 Newberry, Trans. Farad. Soc, 15, p. $126 ; 1919$.

1 Knobel, Caplan and Eiseman, Trans. Am. Electrochem. Soc., 43, p. 55; 1923. Tartar and Keyes, Jour. Am. Chem. Soc., 44, p. 557; 1922. McInnes, Jour. Am. Chem. Soc., 42, p. $2233 ; 1920$.

s LeBlanc., Abhandl. đ. Bunsen Ges., 3; 1910.

- Reicheustein. Zeit. f. Elektrochem., 16, p. 916; 1910.
} 
disappearing quantity, an oscillographic mothod appears to be the only possible one for obtaining a true picture of the process. The application of the electron-tube amplifier removes the above objection to the use of the oscillograph for the study of polarization as will be apparent from the following discussion.

In the case of an electron tube, the plate current supplied by the battery, $B$, is controlled by the potential of the grid with respect to the filament. (See fig. 1.) If the grid be maintained sufficiently negative with respect to the filament as it is with the battery, $C$, no electrons will flow to the grid. Under this condition the tube absorbs no power in the grid circuit. The grid will act simply as a control electrode and the relation between the voltage $C$ and the corresponding plate current will, in general, be that shown in the curve in Figure 2. Over. a portion of this curve the relation between grid potential and plate current is approximately linear.

If now the cell under investigation be put in series with the grid battery of constant voltage and their sum be kept between $V_{1}$ and $V_{2}$; that is, within the limits for which the graph is a straight line, then any variation in the potential of the cell will be truly represented by the corresponoding variation of the plate current within the limits $I_{1}$ and $I_{2}$. An oscillographic element may, therefore, be used in the plate circuit to observe and

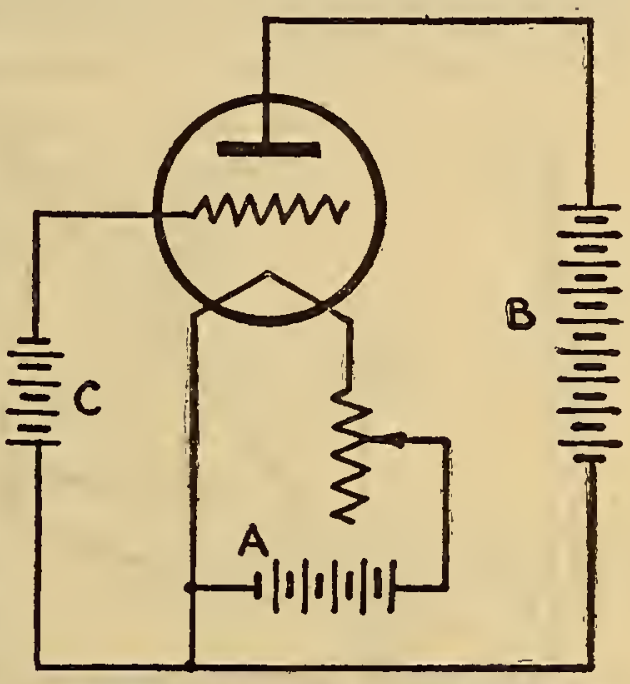

FIg. 1.-Eleotron tube with batteries photographically record the variations in potential of the cell without requiring any current whatever from the cell, the power to operate the oscillograph being furnished by battery $B$.

If a direct current be passed through the cell and suddenly interrupted and the variation in plate current be simultaneously photographed, a record of the polarization of the cell may be obtained. Likewise, the effect of polarization in opposing the applied voltage immediately after closing the direct-current circuit may also be recorded photographically. The use of the electron tube and oscillograph, therefore, furnishes a means of obtaining a true record of the transient phenomenon of polarization. This is true for the reasons that (1) when the direct current is interrupted, the potential difference due to resistance instantly disappears and no error is possible from that source, and (2) the potential difference across 
the cell is not affected in any possible way by the apparatus used in the observation.

If an alternating current be passed through the cell, and if the sum of the voltages of the grid battery $C$ and the cell (that is, the total grid voltage) be kept near the middle of the graph as at $X$, then an alternating component will be obtained in the current of the plate circuit. So long as the amplitude, $E_{\max }$, of the alternating potential across the cell does not exceed the limits $V_{1}$ and $V_{2}$, between which the graph is a straight line, then the alternating component in the plate circuit is a true representation of the alternating potential across the cell. If this alternating component in the plate circuit be measured, the value of the alternating potential across the cell may then be obtained directly from the graph in Figure 2. Thus the electron tube furnishes means of measuring the true al-

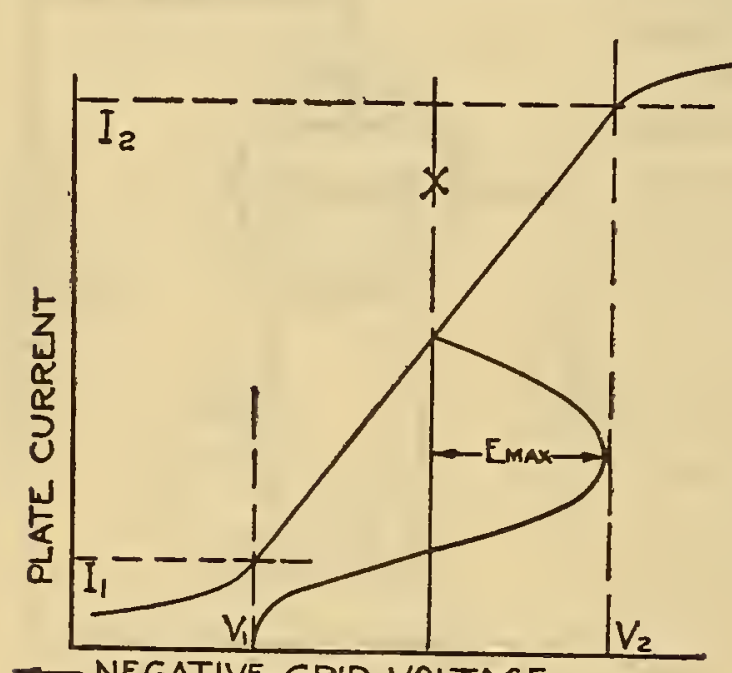

- NEGATIVE GRID VOLTAGE

FIG. 2.-Relation between grid voltage and plate current

ternating potential difference across the cell without affecting the cell. This affords a means of computing the impedance and also the resistance of the cell independently of its electromotive force.

If a half cell of constant electromotive force be brought in electrolytic contact with the cell, each electrode of the cell may be studied separately by the methods outlined. Thus, in the present work, an acidified mercurous-sulphate half cell was used, and the electrodes studied were in sulphuric acid of the same density (about 1.25). Since, both in the observations on polarization and also in the measurement of the alternating potential difference, the readings were independent of the electromotive force of the half cell, its value was of no consequence except that it was necessary that it be constant. It was used only as a means of making electrolytic contact with the cell. The results given below refer to the separate electrode in each case.

This paper aims to present a description of the method and illustrations of its use (1) in conjunction with the oscillograph for observing and recording variations in the potential of an electrode with changing conditions, and (2) for observing the potential and current relations with an alternating current passing through the electrode. Since the work was temporarily interrupted, insufficient data were 
obtained to interpret all the results obtained on the various electrodes, but it is believed that sufficient evidence was obtained to demonstrate the usefulness of the method in studying polarization, overvoltage, decomposition voltage, and other electrode phenomena.

\section{DESCRIPTION OF THE AMPLIFIER AND ITS USE WITH THE OSCILLOGRAPH}

With only one tube as in Figure 1, the plate current was not sufficient to operate the oscillograph, which required a current of about 150 milliamperes for full-scale deflection. To obtain a sufficient plate current to operate the oscillograph and obtain minimum distortion, a three-tube resistance-coupled amplifier was used (fig. 3) at the suggestion of Dr. C. B. Jolliffe, of this bureau. It was similar to that described by Van der Bijl ${ }^{7}$ and King ${ }^{8}$ and consisted of three power tubes coupled by noninductive resistances $R_{1}$ and $R_{2}$ of about 50,000

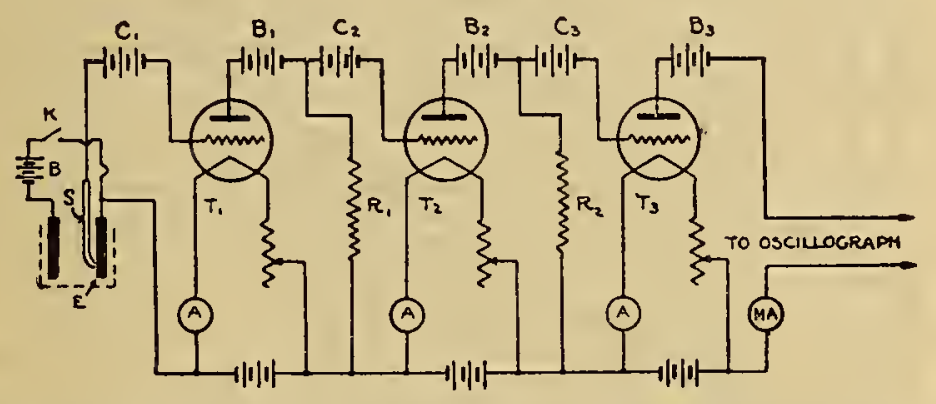

FIG. 3.-The resistance-coupled amplifier as used with the oscillograph and auxiliary electrode, $S$, for studying the potential of the electrode, $E$

ohms each. $T_{1}$ and $T_{2}$ were 5-watt tubes and $T_{3}$ was a 50-watt tube. The filament currents were kept constant, as indicated by the ammeters at $A$. The plate batteries $B_{1}$ and $B_{2}$ were about 100 volts each, and $B_{3}$ was about 500 volts. The grids were maintained negative by the batteries $C_{1}, C_{2}$, and $C_{3}$, the latter two being about 40 volts each. The voltage of $C_{1}$ was varied over the range in voltage necessary to give the desired variation in plate current as measured by the milliameter $M A$. The relation between the negative grid voltage of $C_{1}$ and the plate current of the third tube, $T_{3}$, is shown in Figure 4 . Over the range in voltage used (3.4 to 4.1 volts) the graph is practically a straight line. As long as the variation in grid voltage did not exceed this range in voltage the variation in plate current (30 to 70 milliamperes) as observed by means of the oscillograph was a true representation of the varying grid voltage.

7 Van der Bijl., The Thermionic Vacuum Tube and its Applications, p. 259 ; 1920.

${ }^{8}$ King, Jour. Opt. Soc., 8, p. $98 ; 1924$. 
The cell consisting of the mercurous-sulphate electrode $S$ and the electrode $E$ under investigation was placed in series with the grid battery $C_{1}$. The voltage of the cell was measured with a potentiometer. The voltage of the grid battery $C_{1}$ was indicated by a voltmeter, and its value was adjusted by means of a rheostat, until the sum of its voltage and that of the cell under investigation came within the range of voltage required by the characteristic graph in Figure 4. For example, when the voltage of the cell was 1 volt, and it was desired to work at a grid voltage of 3.80 volts, the voltage of $C_{1}$ was adjusted to 2.80 volts.

The tip of the half cell was kept in contact with the front surface of the electrode to be studied, in order to reduce to a minimum the potential difference due to the resistance of the electrolyte between the tip of the half cell and the electrode. Direct current was passed

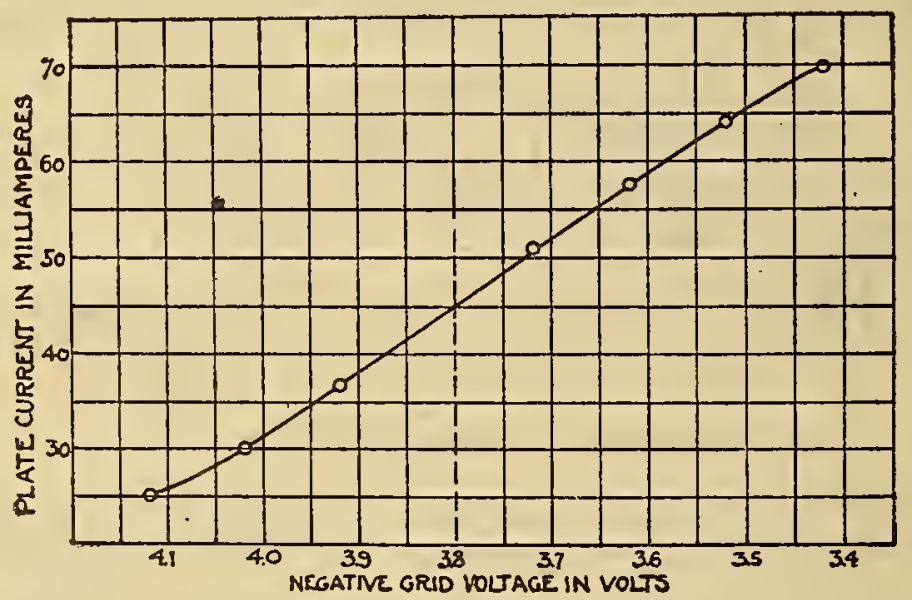

FIG. 4.-Graph showing linear relation between voltage on grid of $T_{1}$ (fig. 3) and plate current of $T_{3}$

through the cell by means of the storage battery, $B$, and this direct current circuit was closed and interrupted by means of switch, $K$.

In order to obtain an oscillographic record of the polarization, the switch, $K$, was closed and opened during the photographic exposure of the plate current in $T_{3}$. Several illustrations of the records of polarization obtained by this method are shown in Figures $6,7,8$, and 9. Thus, Figure 6 represents the polarization of a platinized platinum anode when the direct-current circuit was closed and opened by switch, $K$, at the instants of time represented by the 60 -cycle alternating current on the same film. Thus, the switch was closed for an interval of about 0.50 second. The electrode area was about $3.5 \mathrm{~cm}^{2}$ and the current about 0.5 ampere so that gas was evolved freely. When the switch was closed the polarization opposed the impressed voltage and an interval of about 0.13 second was required for the electrode potential to reach a constant value. 
It should be noted both at the instant of closing and opening the circuit that the potential changed instantaneously. Since any potential difference required for the current to overcome the resistance must disappear instantly when the current becomes zero, this instantaneous change was doubtless due to resistance. On opening the circuit, the polarization decreased comparatively slowly after the sudden drop in potential due to resistance. A small part of this resistance was due to the resistance of the platinum wire leading to the electrode. It appears from these oscillograms, therefore, that the difference between the electrode potentials with and without current flowing, was partly due to an electromotive force and partly due to resistance. Furthermore, in such a case as this where polarization disappears slowly, the commutator method would give a fairly satisfactory measure of polarization. On the other hand, measurement of polarization by the direct method with the potentiometer during a continuous current would give a result too high by an amount equal to the product of the current and the resistance.

The oscillographic record in Figure 7 shows the closing and opening of the circuit under the same conditions as existed in obtaining Figure 6, except that the platinum was not platinized. It is at once apparent that the polarization either disappeared so quickly that its magnitude could not be estimated with any degree of accuracy, or that it was very small. If the polarization was as small as the record indicates then the potential difference due to resistance was much greater than it was in the case of platinized platinum. In this case the direct method would obviously not be satisfactory for measuring polarization because of the large error due to resistance. The results with the commutator method would also be doubtful in this case because of the rapid depolarization or the small value of the polarization as the case may be. The effect of platinizing the electrode is clearly shown by comparison of Figures 6 and 7 .

The oscillographic record in Figure 8 shows the variation in potential during the closing and opening of the circuit in the case of a small negative (lead) storage battery plate of the pasted type (43 by $14 \mathrm{~mm}$ ) when used as a cathode with a current of 0.5 ampere. The oscillogram in Figure 9 shows the result for the same plate when used as anode under otherwise the same conditions as existed in the case of Figure 8. Since there is no instantaneous change in the potential, but a very gradual change from the instant of closing and of opening the circuit, there is no evidence in the oscillogram of any part of the potential being due to resistance, as there was in the case of the platinum electrodes shown in Figures 6 and 7 .

From these illustrations it appears possible to distinguish between electromotive force of polarization and potential difference due to resistance by means of the amplifier and oscillograph, particularly in 
the case where the polarization does not disappear too rapidly. The evidence in these oscillograms of the presence or absence of resistance is confirmed by the actual measurement of the electrode resistance according to the method described below.

\section{USE OF THE AMPLIFIER IN THE MEASUREMENT OF THE RESISTANCE AT AN ELECTRODE}

The measurement of resistance at an electrode is complicated by the problem of distinguishing between electromotive force and potential difference due to resistance. As pointed out previously, the oscillograph is helpful in making this distinction particularly in the case where the polarization does not disappear too quickly. It does not seem that any direct-current method of measurement is applicable to this problem. However, if an alternating current is passed through the cell, the alternating component of the total volt-

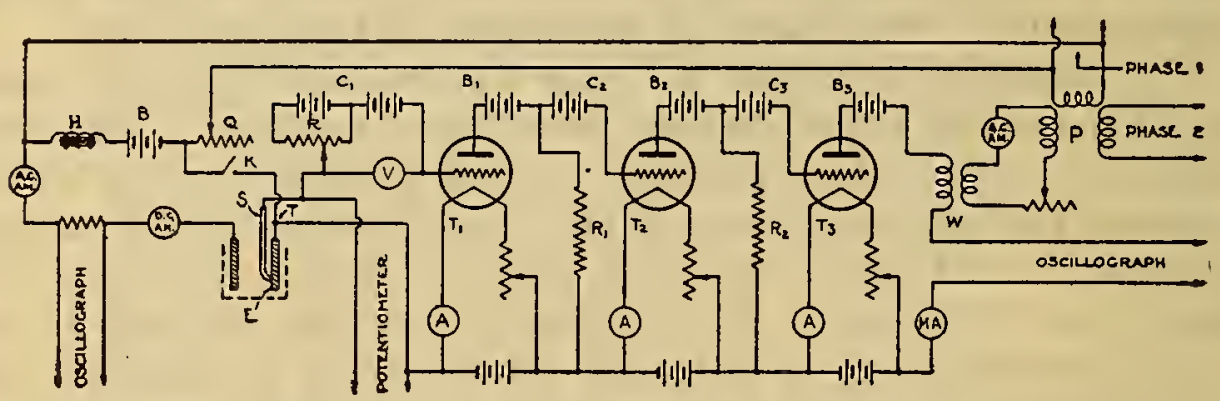

F'I. 5.-The resistance-coupled amplifier and auxiliary circuits

The electrode $E$ under investigation and the half-cell $S$ in electrolyte constitute a cell in series with the variable grid battery $C_{1}$. An oscillograph element and the voltage coil of a wattmeter are in series in the plate circuit of the tube $T_{3}$. Phase 1 from a two-phase generator supplies alternating current to the cell containing $E$, and phase 2 excites the current coil of the wattmeter. The phase shifter at $P^{\prime}$ is used to bring the currents through the wattmeter in phase. Battery $B$ supplies direct current to $E$

age across the cell should be entirely independent of the electromotive force of polarization. If it were possible to obtain the true alternating potential difference across the electrode, then the resistance may be estimated from the current and the potential provided the phase relations are known. The amplifier and half cell supplies a ready means of measuring the alternating potential difference across the electrode without requiring any power from either the half cell or the electrode under investigation. The true alternating potential difference is thereby obtained. This method of measurement was, therefore, tried, and its description follows.

The alternating current was passed through the cell containing the electrode, $E$, under investigation, being supplied by a two-phase generator (fig. 5). The choke coil, $H$, was used to prevent most of the alternating current from flowing through the battery, $B$. The current was adjusted by the rheostat, $Q$. One element of the oscil- 


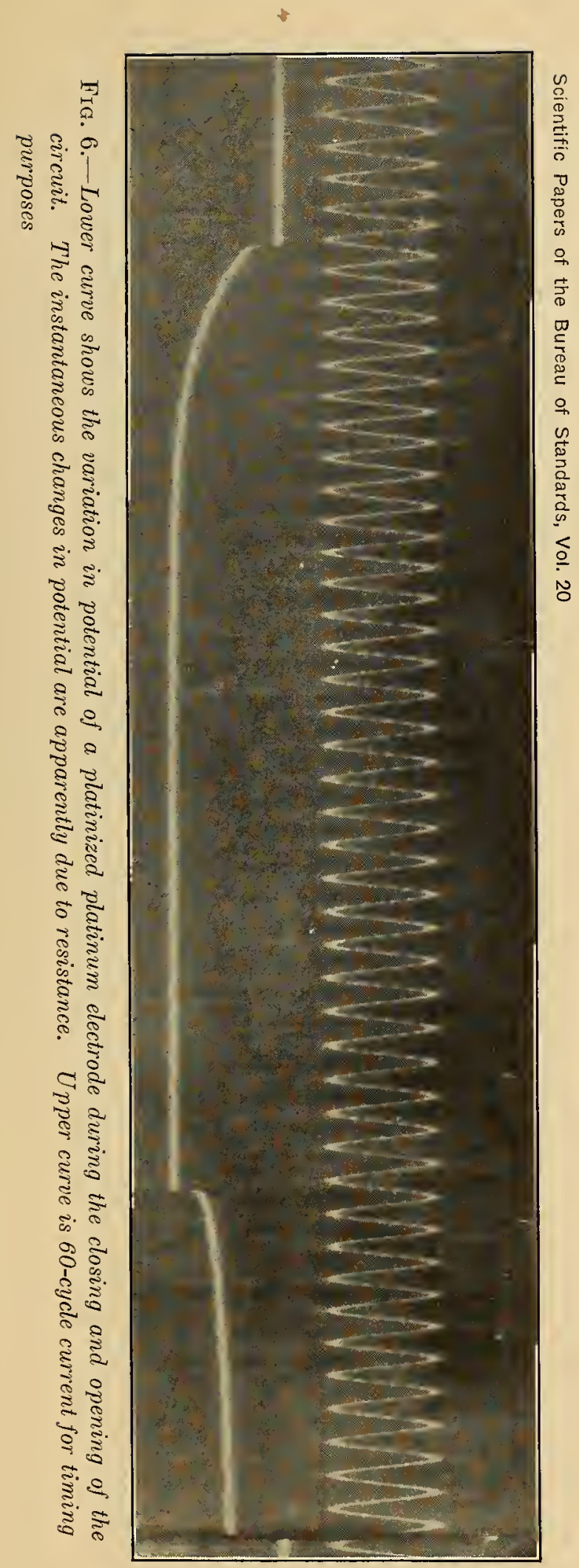




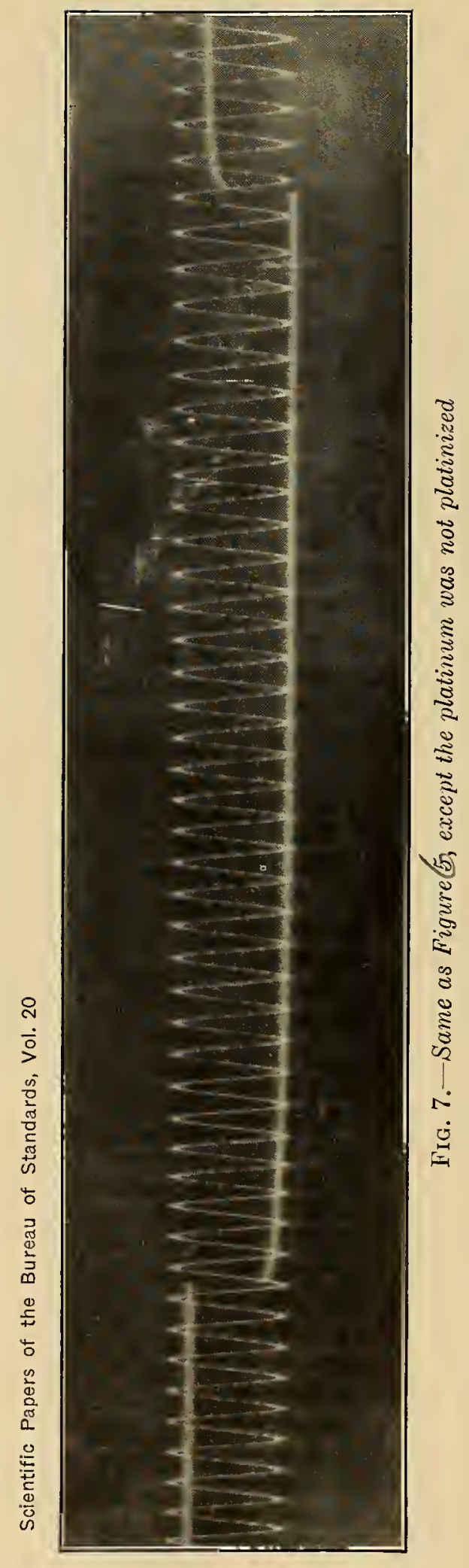




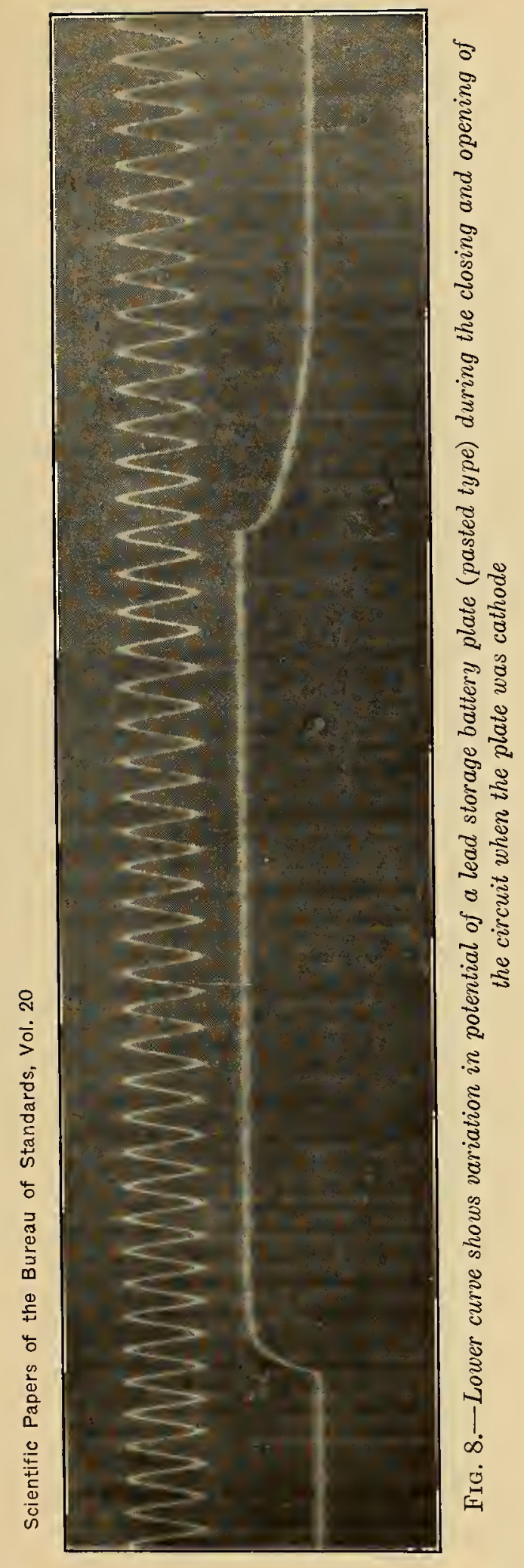




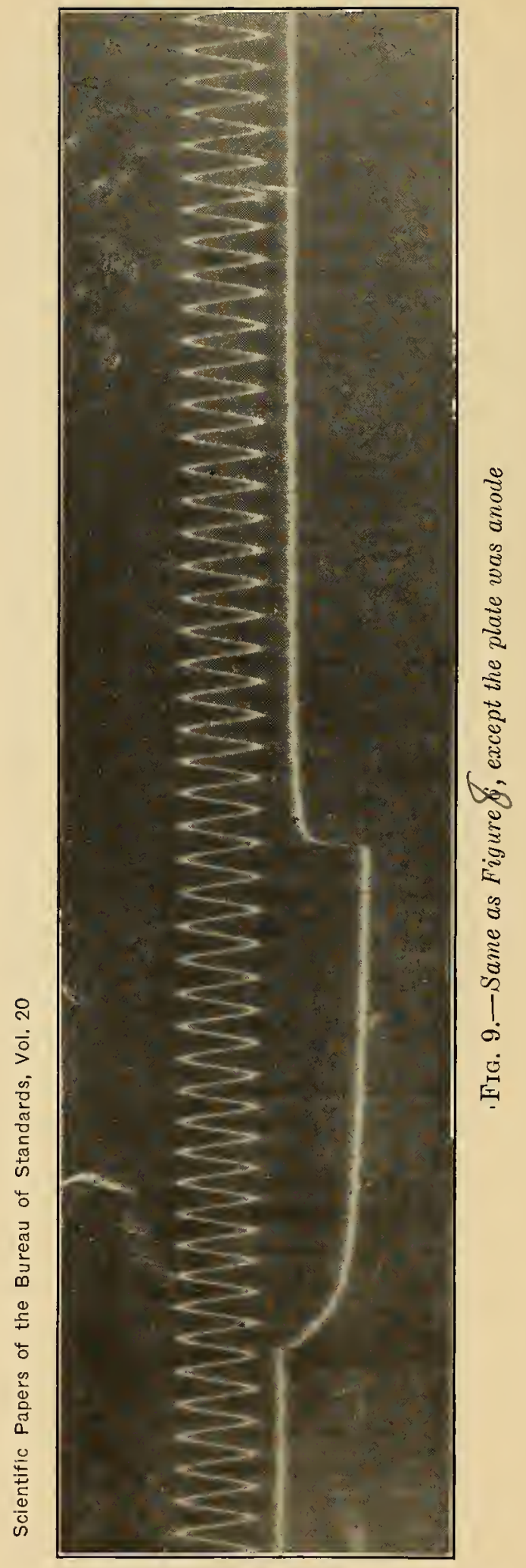


lograph was connected across a resistance unit in series with the cell under investigation in order to observe the wave form of the current through the cell. For the measurement of the alternatingcurrent component in the plate circuit of the tube $T_{3}$, several methods might have been used. The most convenient one appeared to be the use of a wattmeter, the reading of which in this case was entirely independent of the direct-current component. The current to be measured was passed through the voltage coil of the wattmeter, the current coil being excited by a separate source. This source was a separate phase of the same generator as was used for supplying current to the cell. The exciting current was kept constant and was brought in phase with the plate current by means of the phase shifter, $P$. Under these conditions the wattmeter gave a maximum reading (power factor $=1$ ).

The current $I_{1}$ through the cell was maintained at 0.50 ampere (root mean square value) and the current $I_{2}$ through the current coil of the wattmeter was 1.50 amperes. Then the alternating plate current $I_{3}$ is given by the equation

$$
I_{3}^{\prime} \div \frac{W}{I_{2} r}
$$

where $W=$ wattmeter reading and $r=$ resistance of the voltage coil. The alternating potential difference $E_{\mathrm{c}}$ across the cell may then be obtained from the graph in Figure 4. Thus, if $K=\frac{\text { grid potential }}{\text { plate current }}=\frac{4.02-3.42 \text { (volts })}{70-30 \text { (milliamperes })}=\underset{\text { pere }}{0.0150 \text { volts per milliam- }}$ then

$$
E_{\mathrm{c}}=K I_{3}=0.015 I_{3}
$$

In order to check up the reliability of this method of measurement, a known resistance $(0.32 \mathrm{ohm})$ was inserted in series with the grid battery $C_{1}$. The potential difference across this known resistance was computed from the values of $I_{3}$ when an alternating current of 0.50 ampere was passed through the resistance. The grid voltage was kept at 3.80 volts, the plate current being 45 milliamperes (d. c.); that is, at the middle of the curve (fig. 4). The agreement between the measured and computed value of the potential difference was satisfactory; that is, within one or two divisions on the wattmeter scale. With the currents used, the deflection of one division of the wattmeter was equivalent to a resistance of $0.0054 \mathrm{ohm}$.

The oscillogram (fig. 10) showed that the alternating current in the plate circuit was in phase with the current through the electrode under investigation, the capacity effect being negligibly small. It was similarly shown that the amplifier itself produced no appreciable change in phase. Under these conditions the resistance of the electrode could be computed by Ohm's law from the current through it and the potential as measured with the amplifier and wattmeter. 
The results of some of the resistance measurements are given in two ways. First, in Figure 11, the resistance as computed from the potential difference across the electrode and the current through it is plotted against the direct current. Second, the potential difference due to resistance (product of direct current and resistance) is plotted against the direct current (figs. 12, 13, and 14).

The results in Figure 11 show that the resistance between the tip of the half cell and the terminal $T$ of the electroder $E$ under investigation was of a readily measurable magnitude in some cases. Except in the case of platinized platinum the resistance varied widely with the current, tending to decrease decidedly as the current was increased. The resistance of the platinum electrode whether smooth or

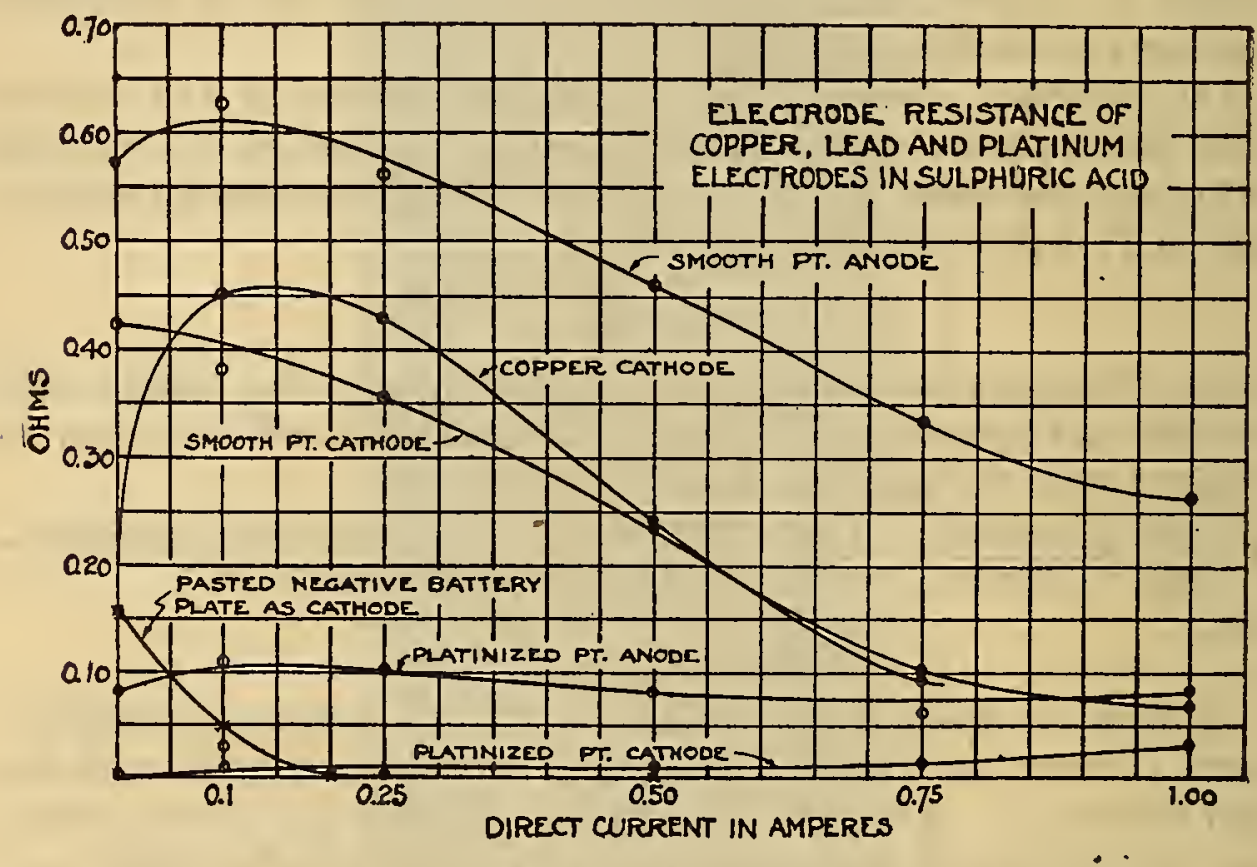

Frg. 11.-Variation of the electrode resistance with direct current

platinized was greater when oxygen was liberated than when hydrogen was liberated on its surface. The resistance of the platinized platinum when used as cathode was almost negligible. The resistances were somewhat variable, but their relative values were fairly reproducible. Thus, in several series of measurements it was always found that the resistance of a smooth platinum electrode was much greater than that for platinized platinum. The results for platinum confirm the conclusions from the oscillograms in Figures 6 and 7 ; that is, that a much greater resistance was present in the case of the smooth platinum specimen than in that of the platinized platinum. In the case of a pasted storage battery plate (lead negative) the resistance was negligible after the passage of an initial current. This confirms the oscillogram in Figures 8 and 9 ; that is, that the poten- 
Scientific Papers of the Bureau of Standards, Vol. 20

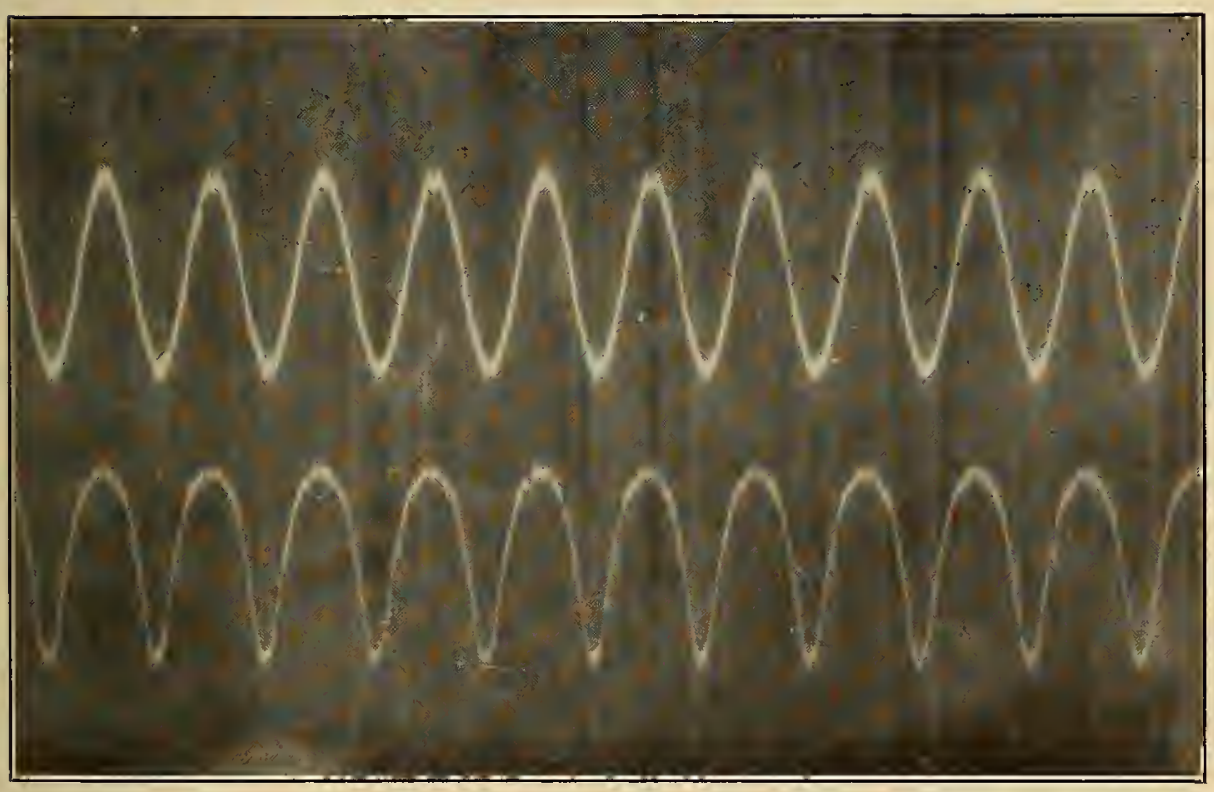

FIG. 10.-Lower curve represents plate current and upper curve represents current through the cell 
tial difference due to resistance was negligibly small in comparison with the polarization. The resistance of a copper electrode in copper

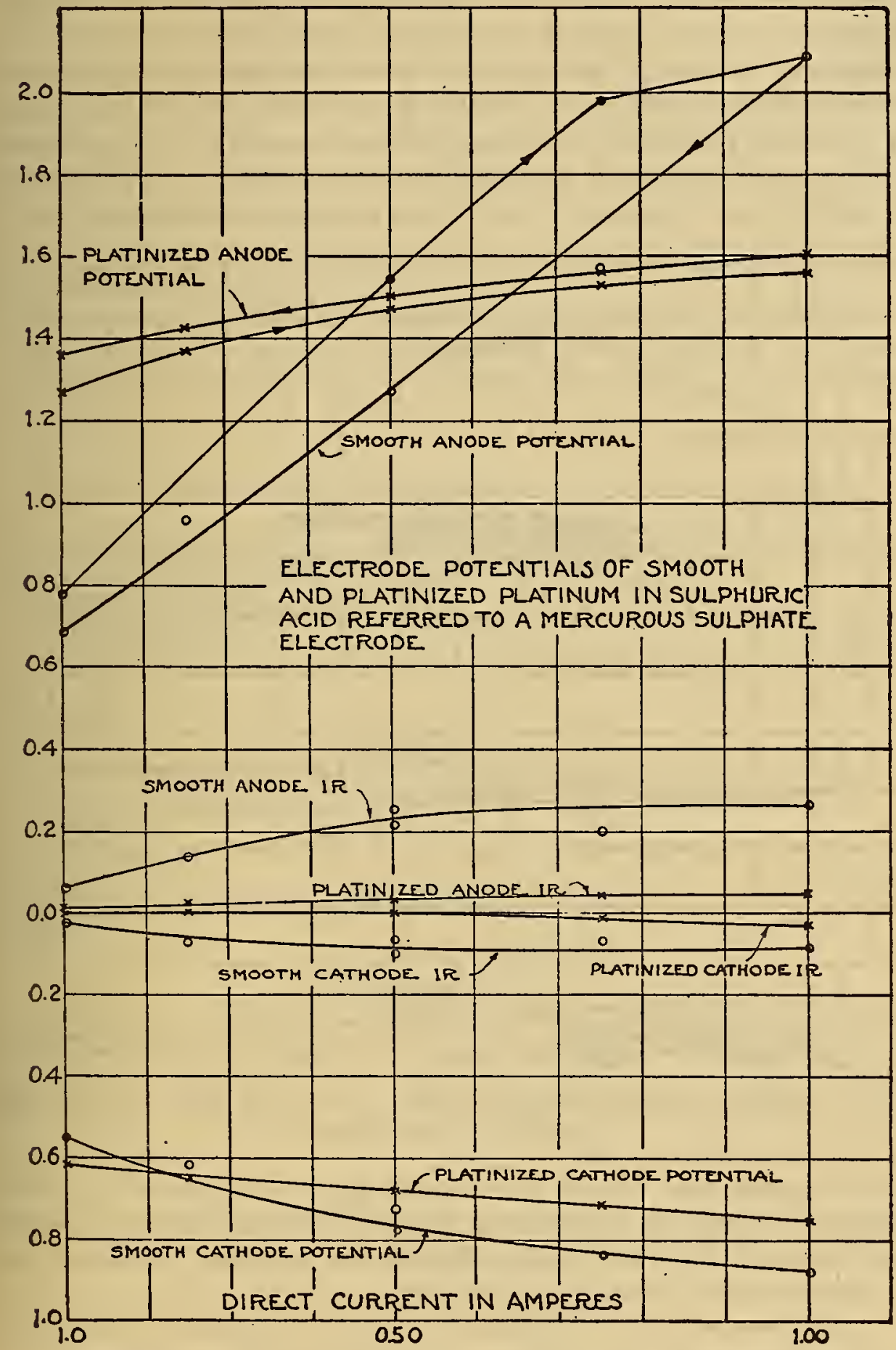

FIG. 12.-Relation between the potential difference due to resistance and the electrode potential as measured by a potentiometer

sulphate solution and of a zinc electrode in zinc sulphate solution was almost negligible. 
In Figure 12 the potential difference due to resistance, and also the electrode potential referred to the mercury electrode, are plotted against the current. The wide variation of the potential of a smooth platinum anode is shown by the curve for increasing and also for decreasing current. The potential difference due to resistance of the smooth anode varied much less than, but in the same direction as, the anode potential. This suggests that a small part of the variation in potential was due to variation in the resistance. In this case, as much as two-tenths of a volt of the potential as measured by the

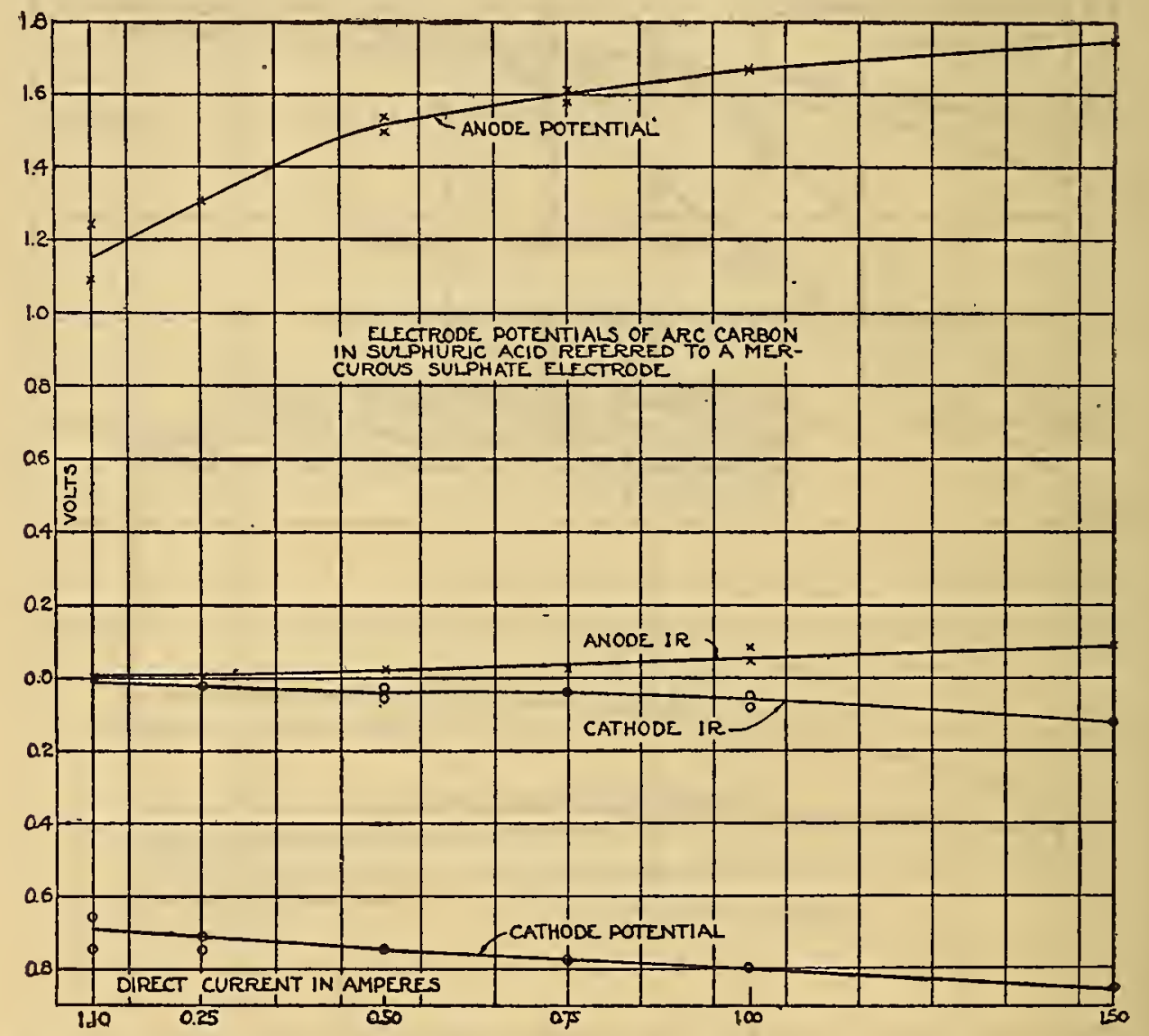

FIG. 13.-Relation between potential difference due to resistance and electrode potential of aro carbon

potentiometer was due to resistance. In the case of the smooth platinum cathode and platinized anode the errors due to resistance were less and for platinized platinum the potential difference due to resistance was practically negligible.

In the case of carbon electrodes (fig. 13) the potential due to resistance was small, and it bears practically a linear relation to the current; that is, the resistance was nearly constant.

In the case of a copper electrode in a copper sulpliate solution acidified with sulphuric acid, the conditions were more complicated 
(fig. 14). When it was anode, the electrode resistance was very small and practically constant. When the copper was cathode the resistance was low until the current was sufficient to liberate hydrogen rapidly. Then both the total potential and resistance increased rapidly. With this rapid evolution of hydrogen, the deposit became spongy and both the electrode potential and resistance dropped appreciably. On reducing the direct current, resistance disappeared almost entirely and the cathode potential returned to its initial value. These curves show that part of the potential as measured with a potentiometer during the evolution of gas was due to resistance. For instance, at 1 ampere the total cathode potential was about 0.65 volt, of which about 0.15 volt was due to resistance.

From these observations it appears that the boundary resistance was appreciable only when gas was being liberated upon the surface

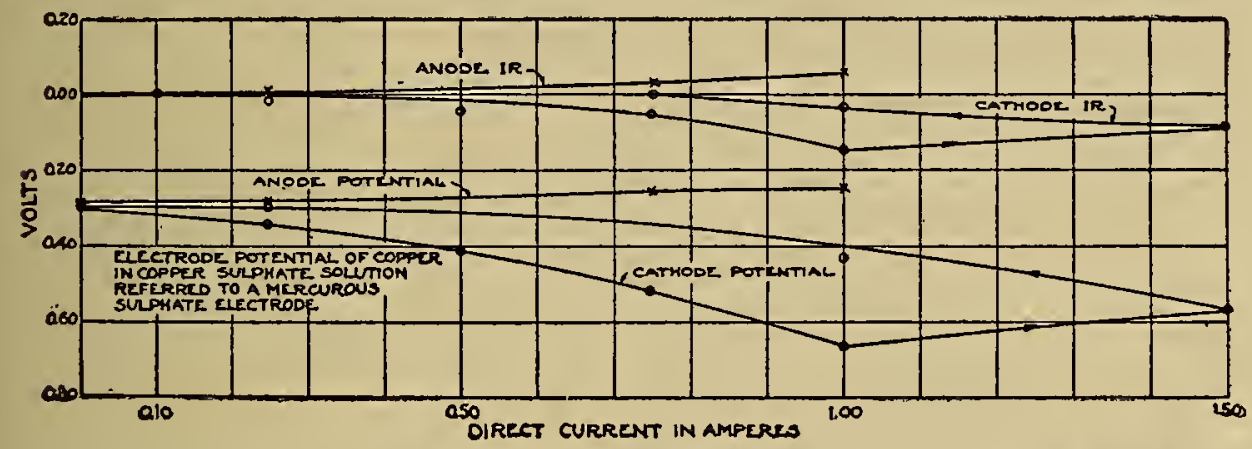

FIr. 14.-Variation in potentiai due to resistance and also variation in electrode potential with current in the case of copper in copper sulphate solution

of the electrode. If this is confirmed by further experiments, the boundary resistance is probably due to a poorly conducting film of gas.

\section{CONCLUSIONS}

A resistance-coupled electron tube amplifier was used to operate an oscillograph for observing the variations in the potential of an electrode without requiring any current from the electrode under investigation. By making oscillographic photograplss of the electrode potential at the instant of interrupting a direct current through the cell under investigation it was possible to obtain a continuous record of the variation in potential. In cases where the polarization disappeared slowly enough, distinction could be made between the electromotive force of polarization and potential difference due to resistance.

By superposing alternating current upon a cell consisting of the electrode under investigation and the half cell, it was possible with the use of the amplifier and a separately excited wattmeter to measure the true alternating potential difference due to resistance at the 
electrode. From this potential difference and the current through the cell, the resistance was computed from Ohm's law. This was permissible because the potential and current, as shown by the oscillograph, were in phase, there being a negligibly small capacity effect.

The resistance varied considerably with the different kinds of electrodes and with the current. The resistance of platinized platinum, lead, and carbon in sulphuric acid was small; that is, several hundredths of an ohm, while that of smooth platinum and copper in the same electrolyte was comparatively high, several tenths of an $\mathrm{ohm}$. The resistance of copper in copper sulphate and zinc in zinc sulphate was very small. The results show that in some cases the electrode potential as measured by the potentiometer when current is flowing is more than 0.2 volt too high because of the potential drop due to resistance. In the cases where the resistance was high it decreased rapidly as the current was increased.

Washington, August 22, 1924. 